\title{
Electrical conductivity for radio-frequency fields in strongly magnetized plasmas with density fluctuations
}

\author{
By YU.S.SAYASOV AND CH. P. RITZ \\ Department of Physics, University of Fribourg, CH-1700 Fribourg, Switzerland
}

(Received 16 August 1982 and in revised form 29 October 1982)

A general investigation of the electrical conductivity for radio-frequency (RF) fields in strongly magnetized plasmas with small-scale density fluctuations is performed within the cold plasma hydrodynamical approximation. It is shown that in such plasmas an RF phenomenon similar to the Bohm diffusion exists: the presence of stochastic RF electric field in a turbulent plasma can lead to a strong enhancement of the RF currents flowing in the direction of the applied electric field components transverse to the magnetic field. The appearance of these turbulent drift currents favours energy transfer from the RF fields to the plasma and thus leads to their stronger damping. This effect allows us to interpret quantitatively the enhanced damping of the magnetosonic waves observed in several experiments. The magnetized radially inhomogeneous cylindrical plasmas in these experiments are characterized by density fluctuations due to drift instabilities. The theory has also a number of other applications; an example is given of the whistlers damped by the ionospheric density fluctuations.

\section{Introduction}

The mechanism of electrical conductivity in strongly magnetized plasmas with density fluctuations involves an important feature (similar to Bohm diffusion) which was first discussed for static electrical fields by Yoshikawa \& Rose (1962) and by Kadomtsev (1965). Qualitatively their arguments can be presented as follows. Suppose a homogeneous static magnetic field $\mathbf{B}_{\mathbf{0}}=\left(0,0, B_{0}\right)$ and an electric field $\mathbf{E}=\left(0, E_{y}, 0\right)$ are applied to a homogeneous quiescent plasma of density $\bar{n}$ (figure 1). If the plasma is strongly magnetized, i.e.

$$
\omega_{c e} \gg \nu_{e}
$$

( $\omega_{c e}=e B_{0} / m_{e} c$ is the electron cyclotron frequency, $\nu_{e}$ is the electron collision frequency, $e>0$ ), the electrons perform mainly drift motion directed along the $x$ axis and the corresponding drift current is $j_{x}^{0}=-e \bar{n} v_{d x}, v_{d x}=c E_{y} / B_{0}$. Only a small fraction of this current transforms owing to collisions into the current $j_{y}^{0}=j_{x}^{0} \nu_{e} / \omega_{c e}$ directed along the $y$ axis, i.e. along the applied electric field $E_{y}$. The situation can, however, change substantially if the electron density possesses a small fluctuating part $n^{\prime}$, the spatial variation of the electron density being given by $n(\mathbf{r})=\bar{n}(\mathbf{r})+n^{\prime}(\mathbf{r})$. The applied electric field cannot remain homogeneous, and new random field components such as $E_{x}^{\prime}$ arise in this case. The crossed electric 


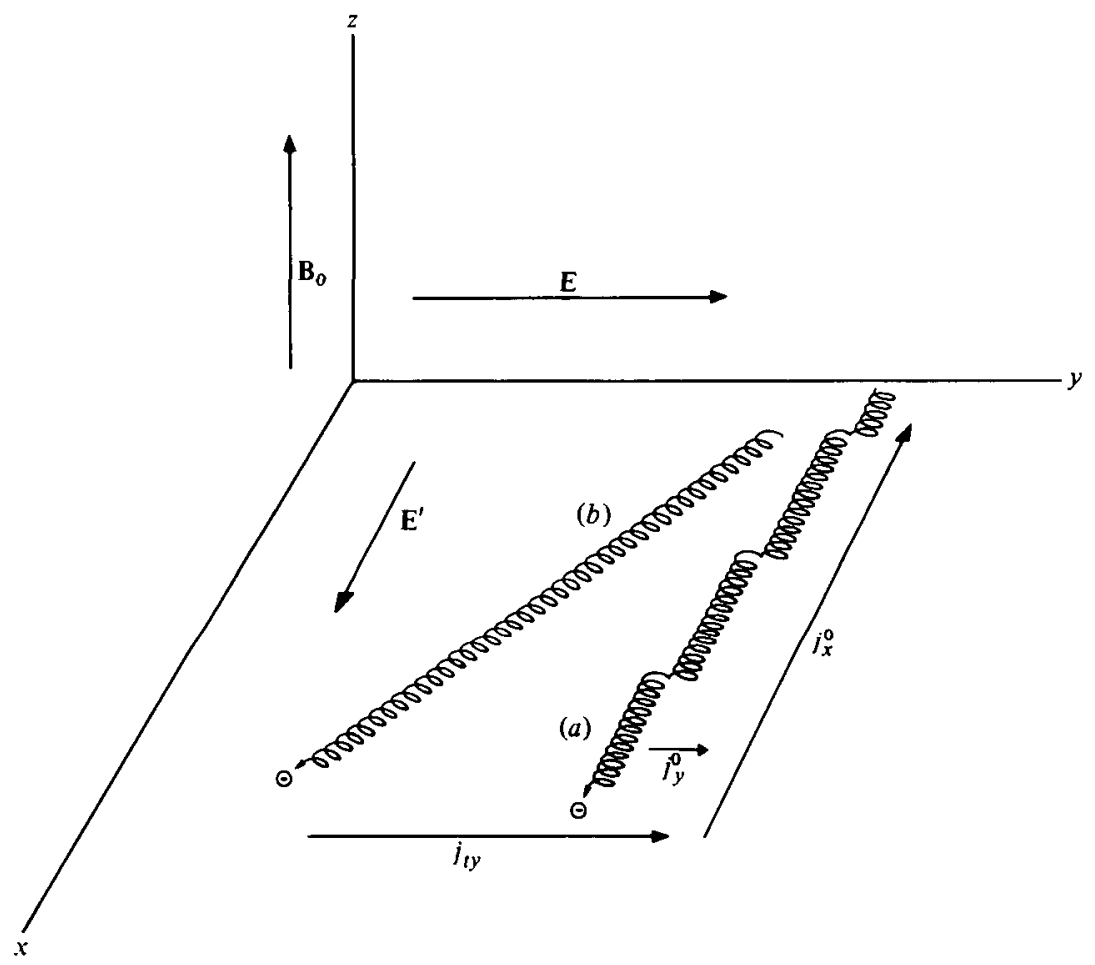

Fraure 1. Schematic representation of the electron motion: (a) without density fluctuation, (b) with density fluctuation. $j_{x}^{0}$, electron current due to $\mathbf{E} \times \mathbf{B}_{0}$ drift; $j_{y}^{0}$, electron current due to collisions; $j_{t y}$, turbulent drift current.

$E_{x}^{\prime}$ and magnetic $\mathbf{B}_{0}$ fields provoke an electron drift motion along the $y$ axis with drift velocity $v_{d y}^{\prime}=c E_{x}^{\prime} / B_{0}$. The resulting turbulent drift current can be expressed as

$$
j_{t y}=-\overline{e\left(\bar{n}+n^{\prime}\right) v_{d y}^{\prime}}=-e c \overline{\left(n^{\prime} E_{x}^{\prime}\right)} / B_{0} .
$$

(The bar means an averaging over the spatially fluctuating quantities. The term $e \bar{n} v_{d y}^{\prime}$ in (1) disappears in this averaging.) Using for the fluctuating field $E_{x}^{\prime}$ a plausible estimate $E_{x}^{\prime} \simeq E_{y} n^{\prime} / \bar{n}$ and inserting it into (1), we get

$$
j_{t y}=-e \bar{n} \overline{\left(n^{\prime} / \bar{n}\right)^{2}} c E_{y} / B_{0}=j_{x}^{0}(\Delta n / \bar{n})^{2} .
$$

Accordingly, the ratio of the total current $j_{y}=j_{y}^{0}+j_{t y}$, flowing along the applied field $E_{y}$, to the current $j_{y}^{0}$ in the quiescent plasma is

$$
\frac{j_{y}}{j_{y}^{0}}=\frac{\nu_{\text {eff }}}{\nu_{e}}=1+\left(\frac{\Delta n}{\bar{n}}\right)^{2} \frac{\omega_{c e}}{\nu_{e}} .
$$

Here $(\Delta n / \bar{n})^{2}=\overline{\left(n^{\prime} / \bar{n}\right)^{2}}$ is the mean square of the relative density fluctuation which we will assume to be small. The quantity $\nu_{\text {eff }}=\nu_{e}+(\Delta n / \bar{n})^{2} \omega_{c e}$ is the effective collision frequency. The ratio $\nu_{\text {efr }} / \nu_{e}$ can be very large if $\left.(\Delta n) / \bar{n}\right)^{2} \gg \nu_{e} / \omega_{c e}$. Thus, the presence of small density fluctuations in strongly magnetized plasmas can change drastically the mechanism of the static electrical conductivity. 
These considerations allow a natural generalization for RF fields, if the electron cyclotron frequency $\omega_{c e}$ greatly exceeds not only the collision frequency $\nu_{e}$ but also the RF frequency $\omega$. In this case an electron moving in the crossed electric $E_{x}^{\prime}$ and magnetic $B_{0}$ fields performs many loops within the RF period $2 \pi / \omega$. Thus we can retain for the turbulent drift current the same estimate as above. Comparing the turbulent drift current with the amplitude $j_{y}^{0}=j_{x}^{0}\left(\nu_{e}-i \omega\right) / \omega_{c e}$ of the $\mathrm{RF}$ current in the quiescent plasma, we find that the ratio

$$
\left|\frac{j_{v}}{j_{y}^{0}}\right|=\left|\frac{j_{y}^{0}+j_{t y}}{j_{y}^{0}}\right|=1+\left(\frac{\Delta n}{\bar{n}}\right)^{2} \frac{\omega_{c e}}{\left|\nu_{e}-i \omega\right|}
$$

can be very big if $(\Delta n / \bar{n})^{2} \gg\left|\nu_{e}-i \omega\right| / \omega_{c e}$. (These estimates involve an assumption, which is not always correct, that the ions do not influence the electron turbulent drift motion. However, they give the right order of magnitude of the effect.) So the presence of small density fluctuations in a strongly magnetized plasma can change drastically the mechanism of the RF conductivity and, hence, the corresponding RF field distribution. It means that substantial discrepancies can be expected between experiments on RF field propagation in turbulent plasmas and the results of calculations performed without including the density fluctuations.

Such discrepancies were indeed found several times in experiments on the damping of magnetosonic waves (MSW), excited in cylindrical radially inhomogeneous magnetized plasmas. In the papers by Lammers (1974) and Krämer (1975) a strong reduction of the plasma admittance (as compared with that calculated for the quiescent plasma) was observed. Anomalously low resonance amplification of the MSW was further observed by Blackwell \& Cross (1979) and by Hoegger et al. $(1980,1981)$ and Schneider et al. (1980). The latter authors supposed the anomalous damping to be due to the density fluctuations measured in the edge region. As theoretical considerations show (see figure 5 in Timofeev \& Shvilkin (1976)), fluctuations due to drift instabilities must exist under the conditions described by Lammers (1974) and Krämer (1975) too. In experiments made by Ritz et al. (1982) and Vaucher et al. (1983), an investigation of the MSW damping was performed with simultaneous measurement of the plasma turbulence. It was found that the anomalously strong damping of MSW observed in these plasmas correlates with the density fluctuations due to the drift instabilities.

It appears therefore desirable to perform a systematic investigation of such effects by extending the theory formulated in the static case by Yoshikawa \& Rose (1962) and by Kadomtsev (1965) to RF fields. A preliminary attempt of this kind was made by Sayasov (1981), where it was shown that the experiments mentioned above can be interpreted by the fact that the RF conductivity tensor for strongly magnetized plasmas changes substantially, if one accounts for the presence of the small density fluctuations. This conclusion was confirmed by more detailed calculations described in Ritz et al. (1982).

In the following, a derivation of the RF conductivity tensor for strongly magnetized plasmas with density fluctuations is presented $(\$ 2)$ in a more general form than hitherto; see Sayasov (1981) and Ritz et al. (1982). The tensor is then applied to a systematic investigation of the electromagnetic wave (EMW) 
propagation in such plasmas under different conditions. In $\S 3, \mathrm{EMW}$ propagation transverse to the magnetic field $\mathbf{B}_{0}$ is investigated. It is shown that the drastic discrepancies between the theory for the quiescent plasma and the experiments on the damping of the MSW can be attributed to the presence of the density fluctuations. In $\S 4$ our theory is applied to the investigation of EMW propagation parallel to the magnetic field $\mathbf{B}_{\mathbf{0}}$. The particular case of ionospheric whistlers is considered.

\section{Derivation of the RF conductivity tensor for strongly magnetized plasmas with density fluctuations}

In what follows, we use some basic assumptions mainly concerning the nature of the density fluctuations in the plasma.

(i) The fluctuations are independent of the strength of the applied RF field, i.e. they are considered as pre-existing in the plasma and not affected by the applied RF field.

(ii) The frequency $\omega_{\mathrm{fl}}$ of the density fluctuations is small compared with the RF frequency $\omega$. This allows us to treat the density fluctuations as a random function of the space co-ordinates only.

(iii) Space scales of the density fluctuations are small compared with some effective length pertaining to the RF field. This allows us to consider the fluctuating part of the RF field as being quasi-static (see Appendix A).

(iv) Space scales of the density fluctuations are big compared with the plasma Debye length. This permits the assumption of quasi-neutrality of the plasma. The assumptions (i)-(iv) appear to be satisfied for the plasmas studied in the experiments mentioned above.

In addition it will be assumed that the cold plasma hydrodynamic approximation is valid. It implies some constraints on the electron and ion cyclotron radii, which must be small compared with the relevant plasma dimensions. We will consider a three-component magnetized plasma consisting of electrons (mass $m_{e}$, charge $-e$, density $n$ ), ions (mass $m_{i}$, charge $e$, density $n$ ) and neutrals. However, to simplify the algebra, we will use a quasi two-fluid theory in which the neutrals will be assumed to be at rest. (Their presence will be, however, accounted for via the electron-neutral collision frequency). This assumption is valid if the ion-neutral collision frequency $\nu_{i n}$ is small compared with $\omega, \nu_{i n} \ll \omega$. The conditions $\omega_{\mathrm{fl}} \ll \omega, \nu_{i n} \ll \omega$ mean that we must refrain from considering the limit $\omega \rightarrow 0$. We also mention that in this limit some thermal effects, e.g. the ion viscosity, which we disregarded, may not be negligible; see Yoshikawa \& Rose (1962)). Nonlinear effects will be neglected too; this means that we will neglect both the change in the density fluctuations and in the electron temperature under the influence of the RF fields (Ginzburg 1961, p. 733). This is justified for the small RF powers used in the experiments analysed in $\S 3$. Finally we recall, as stated in the introduction, that we treat only the case for which the electron cyclotron frequency greatly exceeds the collision frequency and the RF frequency $\left(\omega_{c e} \gg \nu_{e}, \omega\right)$. Beyond this frequency range the influence of the density fluctuations on the electrical conductivity remains small. 
The assumption (ii), allowing us to represent the electron density as being time-independent, in the form $n(\mathbf{r})=\bar{n}(\mathbf{r})+n^{\prime}(\mathbf{r})$, means that the fields excited in a plasma by a harmonic EM field of frequency $\omega$, will be harmonic too, i.e. all the quantities considered below must vary with the time as $\exp (-i \omega t)$. The Maxwell equations for the spatially dependent amplitudes $\mathbf{E}, \mathbf{B}$ have accordingly the form

$$
\operatorname{curl} \mathbf{E}=i \frac{\omega}{c} \mathbf{B}, \quad \operatorname{curl} \mathbf{B}=-i \frac{\omega}{c} \mathbf{E}+\frac{4 \pi}{c} \mathbf{j}
$$

These equations will be complemented by the Ohm's law (valid for $\nu_{i n} \ll \omega$ )

where

$$
\left(\nu_{e}-i \omega\right) \mathbf{j}=\frac{e^{2} n}{m_{e}} \mathbf{E}+\frac{i \omega_{h}^{2}}{\omega}[\mathbf{h} \times[\mathbf{j} \times \mathbf{h}]]-\left(\omega_{c e}-\omega_{c i}\right)[\mathbf{j} \times \mathbf{h}],
$$

(see Ginzburg 1961, p. 172).

$$
\omega_{h}^{2}=\omega_{c e} \omega_{c i}, \quad \omega_{c i}=\frac{e B_{0}}{m_{i} c}, \quad \mathbf{h}=\frac{\mathbf{B}_{0}}{B_{0}}
$$

Following Kadomtsev (1965, p. 119), we replace $\mathbf{E}, \mathbf{B}, \mathbf{j}, n$ by the sums of the average quantities $\mathbf{E}, \overline{\mathbf{B}}, \overline{\mathbf{j}}, \bar{n}$ and the random quantities $\mathbf{E}^{\prime}, \mathbf{B}^{\prime}, \mathbf{j}^{\prime}, n^{\prime}$. For the latter we will use the Fourier representations

$$
\mathbf{E}^{\prime}=\sum_{k} \mathbf{E}_{\mathbf{k}}^{\prime} e^{i \mathbf{k} \cdot \mathbf{r}}, \quad \frac{n^{\prime}}{\bar{n}}=\sum_{k} n_{\mathbf{k}}^{\prime} e^{i \mathbf{k} \cdot \mathbf{r}}
$$

Inserting the definitions $\mathbf{E}=\mathbf{E}+\mathbf{E}^{\prime}, \mathbf{B}=\overline{\mathbf{B}}+\mathbf{B}^{\prime}, \mathbf{j}=\overline{\mathbf{j}}+\mathbf{j}^{\prime}, n=\bar{n}+n^{\prime}$ into (3) and (4), we can split (3), (4) into the equations

$$
\begin{gathered}
\operatorname{curl} \mathbf{E}=i \frac{\omega}{c} \overline{\mathbf{B}}, \quad \operatorname{curl} \overline{\mathbf{B}}=-i \frac{\omega}{c} \overline{\mathbf{E}}+\frac{4 \pi}{c} \overline{\mathbf{j}}, \\
\overline{\mathbf{j}}=\sigma_{0} \mathbf{E}=\overline{\mathbf{j}}_{t}+\frac{i \omega_{h}^{2}}{\omega\left(\nu_{e}-i \omega\right)}[\mathbf{h} \times[\overline{\mathbf{j}} \times \mathbf{h}]]-\chi[\overline{\mathbf{j}} \times \mathbf{h}]
\end{gathered}
$$

governing the average quantities and

where

$$
\begin{gathered}
\operatorname{curl} \mathbf{E}^{\prime}=i \frac{\omega}{c} \mathbf{B}^{\prime}, \quad \operatorname{curl} \mathbf{B}^{\prime}=-i \frac{\omega}{c} \mathbf{E}^{\prime}+\frac{4 \pi}{c} \mathbf{j}^{\prime}, \\
\mathbf{j}^{\prime}=\sigma_{0} \mathscr{E}^{\prime}+\mathbf{j}_{t}^{\prime}+\frac{i \omega_{h}^{2}}{\omega\left(\nu_{e}-i \omega\right)}\left[\mathbf{h} \times\left[\mathbf{j}^{\prime} \times \mathbf{h}\right]\right]-\chi\left[\mathbf{j}^{\prime} \times \mathbf{h}\right],
\end{gathered}
$$

$$
\sigma_{0}=\frac{e^{2} \bar{n}}{m_{c}\left(\nu_{e}-i \omega\right)}, \quad \chi=\frac{\omega_{c e}-\omega_{c i}}{\nu_{e}-i \omega}, \quad \mathscr{E}^{\prime}=\mathbf{E}^{\prime}+\frac{n^{\prime}}{\bar{n}} \mathbf{E}
$$

for the fluctuating quantities.

The term $\mathbf{j}_{t}=\overrightarrow{\mathrm{j}}_{t}+\mathbf{j}_{t}^{\prime}=\sigma_{0} \mathbf{E}^{\prime} n^{\prime} / \bar{n}$ represents the turbulent drift current. It can be specified through the Fourier components $\mathrm{E}_{k}^{\prime}, n_{k}^{\prime}$ (equation (5)) as follows:

$$
\mathbf{j}_{t}=\overline{\mathbf{j}}_{t}+\mathbf{j}_{t}^{\prime}=\sigma_{\mathbf{0}}\left(\sum_{\mathbf{k}} n_{-\mathbf{k}}^{\prime} \mathbf{E}_{\mathbf{k}}^{\prime}+\sum_{k_{1} \neq k_{\mathbf{1}}} n_{\mathbf{k}_{\mathbf{1}}}^{\prime} \mathbf{E}_{\mathbf{k}_{\mathbf{2}}}^{\prime} \exp \left[i\left(\mathbf{k}_{\mathbf{1}}+\mathbf{k}_{\mathbf{2}}\right) \cdot \mathbf{r}\right]\right)
$$

The first term in the bracket can be identified as the mean turbulent current

$$
\overline{\mathbf{j}}_{t}=\sigma_{0} \sum_{k} n_{-k}^{\prime} \mathbf{E}_{k}^{\prime}
$$


The neglect of the second term in the bracket $j_{t}^{\prime}$, involving the randomly distributed phases $\left(\mathbf{k}_{1}+\mathbf{k}_{2}\right)$.r, can be justified within the random phase approximation (see, for example, ter Haar 1958; Krall \& Trivelpiece 1973, p. 550). Except for the neglect of this term, the splitting of the equations (3), (4) into the equations (6)-(9) is an exact operation and we can in this way consider strong turbulence effects in the sense that the turbulent drift current $\overline{\mathbf{j}}_{t}$ in (6) does not need to be small.

To calculate explicitly the current $\overline{\mathfrak{j}}_{t}$, we replace the equation curl $\mathbf{E}^{\prime}=i(\omega / c) \mathbf{B}^{\prime}$ in (8) by curl $\mathbf{E}^{\prime}=0$, implying that $\mathbf{E}^{\prime}=-\nabla \phi$, where $\phi^{\prime}=\Sigma_{\mathbf{k}} \phi_{\mathbf{r}}^{\prime} e^{i \mathbf{r} \cdot r}$ is an electrostatic potential (see Appendix A). Accordingly, we can represent the current $\mathbf{j}_{t}$ in (10) by

$$
\mathbf{j}_{t}=-i \sigma_{0} \sum_{\mathbf{k}} \mathbf{k} n_{\mathbf{k}}^{\prime *} \phi_{\mathbf{r}}^{\prime}
$$

(The coefficients $n_{\mathbf{z}}^{\prime}$ possess the property $n_{-\mathbf{z}}^{\prime}=n_{\mathbf{z}}^{\prime *}$, since $n^{\prime}$ is a real quantity.)

A connexion between the Fourier coefficients $n_{\mathbf{k}}^{\prime}$ (considered to be known) and the Fourier coefficients $\phi_{\mathrm{k}}^{\prime}$ will be found now, following Kadomtsev $(1965, \mathrm{p} .122)$. Equation (9) provides the Ohm's relation between the current density $\mathbf{j}^{\prime}$ and the quantity $\mathscr{E}^{\prime}=-\nabla \phi^{\prime}+\left(n^{\prime} / \bar{n}\right) \overline{\mathbf{E}}$ :

$$
\begin{gathered}
\mathbf{j}^{\prime}=\sigma \mathscr{E}^{\prime \prime}, \quad \sigma=\left(\begin{array}{ccc}
\sigma_{1} & \sigma_{2} & 0 \\
-\sigma_{2} & \sigma_{1} & 0 \\
0 & 0 & \sigma_{0}
\end{array}\right), \\
\sigma_{1}=\frac{\omega}{4 \pi i} \frac{\omega_{p e}^{2} Q}{Q^{2}-P^{2}}, \quad \sigma_{2}=\frac{\omega}{4 \pi} \frac{\omega_{p e}^{2} P}{Q^{2}-P^{2}}, \\
Q=\omega_{h}^{2}-\omega\left(\omega+i \nu_{e}\right), \quad P=\left(\omega_{c e}-\omega_{c i}\right) \omega, \quad \omega_{p e}^{2}=4 \pi \bar{n} e^{2} / m_{e} .
\end{gathered}
$$

Inserting (12) into the continuity equation $\operatorname{div} j^{\prime}=0$ (simplified under assumption (iv)) and using the Fourier series (5), we get a relation between $\phi_{\mathbf{k}}^{\prime}$ and $n_{\mathbf{k}}^{\prime}$ :

where

$$
\phi_{\mathbf{r}}^{\prime}=i \frac{\left(\chi_{1} k_{y}-k_{x}\right) \bar{E}_{x}-\left(\chi_{1} k_{x}+k_{y}\right) \bar{E}_{y}-\chi_{2}^{2} k_{z} \bar{E}_{z}}{k_{r}^{2}+\chi_{2}^{2} k_{z}^{2}} n_{\mathbf{x}}^{\prime}=i a_{\beta} \bar{E}_{\beta} n_{\mathbf{r}}^{\prime}
$$

$$
\chi_{1}=\frac{\sigma_{2}}{\sigma_{1}}=i \frac{P}{Q}, \quad \chi_{2}=\frac{\sigma_{0}}{\sigma_{1}}=\frac{P^{2}-Q^{2}}{Q \omega\left(\omega+i \nu_{e}\right)}, \quad k_{r}^{2}=k_{x}^{2}+k_{y}^{2} .
$$

(It is assumed that the $z$ axis coincides with the direction of the magnetic field $\mathbf{B}_{0}$; repeating indices imply summation over $\beta=x, y, z$.)

We now replace the sum over $\mathbf{k}$ in (11) by the integral over components of the $\mathbf{k}$ vector defined in the spherical co-ordinate system as

$$
k_{z}=k \cos \theta, \quad k_{x}=k \sin \theta \cos \phi, \quad k_{y}=k \sin \theta \sin \phi .
$$

In this way we get an explicit expression for the turbulent drift current $\overline{\mathbf{j}}_{t}$ :

$$
\overline{\mathbf{j}}_{t}=\sigma_{0} \int \mathbf{k}\left|n_{\mathbf{k}}^{\prime}\right|^{2} a_{\beta} \bar{E}_{\beta} d \mathbf{k}=\left(\frac{\Delta n}{\bar{n}}\right)^{2} \mathbf{A E}, \quad A_{\alpha \beta}=\int \frac{q(\theta, \phi) k_{\alpha} a_{\beta} d \cos \theta d \phi}{k^{2}\left[1+\left(\chi_{2}^{2}-1\right) \cos ^{2} \theta\right]},
$$

where $\alpha, \beta=x, y, z$ and the coefficients $a_{\beta}$ are defined by (13). In (15) $q(\theta, \phi)$ means the angular distribution of the density fluctuations

$$
\int\left|n_{\mathbf{r}}^{\prime}\right|^{2} k^{2} d k=\left(\frac{\Delta n}{\bar{n}}\right)^{2} q(\theta, \phi), \quad \int q(\theta, \phi) d \cos \theta d \phi=1
$$


We are now able to write down the conductivity tensor $\sigma_{t}$ via the Ohm's law for the average current (7), i.e. $\overline{\mathbf{j}}=\sigma_{t} \mathbf{E}$. Inserting (15) into (7) and introducing the tensor $\sigma^{-1}$ inverse to $\sigma$, we rewrite (7) in the form

$$
\sigma^{-1} \overline{\mathbf{j}}=I \bar{E}+\left(\frac{\Delta n}{\bar{n}}\right)^{2} \mathbf{A E} \text {. }
$$

Here $I$ is the unit tensor. Multiplying this equation from the left by the tensor $\sigma$ we find the Ohm's law $\mathbf{j}=\sigma_{t} \mathbf{E}$ with the conductivity tensor

$$
\boldsymbol{\sigma}_{t}=\sigma+\left(\frac{\Delta n}{\bar{n}}\right)^{2} \boldsymbol{\sigma A}
$$

accounting for the density fluctuations. Note that $\sigma_{t}$ is also valid under conditions when the elements of the tensor $(\Delta n / \bar{n})^{2} \boldsymbol{\sigma A}$ are not small compared with the corresponding elements of the tensor $\sigma$ for the quiescent plasma. This tensor can be used for investigation of various electrodynamical effects provoked in the magnetized plasma by the density fluctuations, for instance via the equation

$$
\operatorname{curl} \operatorname{curl} \mathbf{E}=\frac{\omega^{2}}{c^{2}} \epsilon_{t} \mathbf{E}, \quad \epsilon_{t}=\mathbf{I}+\frac{4 \pi i}{\omega} \sigma_{t}
$$

resulting from (6) and (17).

We now bring the tensor $\epsilon_{t}$ to a workable form. The function $q(\theta, \phi)$ must be invariant relative to an inversion of the direction of the magnetic field $\mathbf{B}_{0}$, i.e. it must allow the property $q(\theta, \phi)=q\left(\frac{1}{2} \pi-\theta, \phi\right)$. As a consequence the terms in the tensor $\mathbf{A}$ containing $k_{x} k_{z}, k_{y} k_{z}$ disappear in the result of the integration over $\theta$. Further simplification can be achieved if the angular distribution of the fluctuations $q(\theta, \phi)$ depends only slightly upon the angle $\phi$, where $\phi$ lies in the plane perpendicular to $\mathbf{B}_{0}$. (The spectra investigated by Ritz et al. (1982) satisfy approximately this requirement.) Then $q(\theta, \phi) \simeq q(\theta)$ and the integrals over $\phi$, containing the products $k_{x} k_{y}$, disappear. From (18) we thus get (the bars indicating averaged values will be dropped in the following):

where

$$
\epsilon_{t}=\left(\begin{array}{ccc}
\epsilon & i g & 0 \\
-i g & \epsilon & 0 \\
0 & 0 & \eta
\end{array}\right)
$$

and

$$
\begin{gathered}
\epsilon=1+\frac{\omega_{p e}^{2} Q}{Q^{2}-P^{2}} A, \quad g=\frac{\omega_{p e}^{2} P}{Q^{2}-P^{2}} \\
A=1+\left(\frac{\Delta n}{n}\right)^{2} \mathscr{I}_{1}\left(\frac{P^{2}}{Q^{2}}+1\right), \quad \eta=1-\frac{\omega_{p e}^{2}}{\omega\left(\omega+i \nu_{e}\right)} B, \quad B=1-\left(\frac{\Delta n}{n}\right)^{2} \mathscr{I}_{2}
\end{gathered}
$$

$$
\mathscr{I}_{1}=\pi \int_{0}^{\pi} \frac{q(\theta) \sin ^{2} \theta d \cos \theta}{1+\left(\chi_{2}^{2}-1\right) \cos ^{2} \theta}, \quad \mathscr{I}_{2}=2 \pi \int_{0}^{\pi} \frac{q(\theta) \chi_{2}^{2} \cos ^{2} \theta d \cos \theta}{1+\left(\chi_{2}^{2}-1\right) \cos ^{2} \theta} .
$$

The off-diagonal elements of the tensor (19) contain in fact some additional terms proportional to $(\Delta n / n)^{2}$. However, these terms always remain very small owing to our basic assumption $(\Delta n / n)^{2} \ll 1$, and for this reason they were omitted. In contrast, the terms containing $(\Delta n / n)^{2}$ in the diagonal elements of (19) must be retained since they are multiplied by a large factor $\mathscr{I}_{1}\left(P^{2} / Q^{2}+1\right)$ (see Appendix B). 
The integrals $(20)$ can be evaluated in a general form if the frequency $\omega$ greatly exceeds the ion cyclotron frequency $\omega_{c i}$. We then have (since $P \gg|Q|$ ) the inequality $\left|\chi_{2}^{2}\right| \simeq\left|P^{2} /\left(Q \omega\left(\omega+i \nu_{e}\right)\right)\right| \simeq\left|\omega_{c e} \omega /\left(\omega_{c i}\left(\omega+i \nu_{e}\right)\right)\right| \gg 1$. (It must, however, be stressed here that, for $\omega \lesssim \omega_{c i},\left|\chi_{2}^{2}\right| \simeq 1$.) Treating $\cos \theta=\mu$ as a complex variable, we can calculate the integral $\mathscr{J}_{1}$ by means of the residue theorem as follows:

where

$$
\mathscr{I}_{1}=\frac{\pi^{2} q\left(\mu_{0}\right)}{\chi_{2}}-\pi \int_{\Gamma} \frac{q(\mu)\left(1-\mu^{2}\right) d \mu}{1+\chi_{2}^{2} \mu^{2}} \simeq \frac{\pi}{4} \frac{I}{\chi_{2}},
$$

$$
\mu_{0}=i / \chi_{2}, \quad I=4 \pi q\left(\mu_{0}\right)
$$

and $\mu_{0}$ is a root of the equation $1+\chi_{2}^{2} \mu^{2}=0$ having $\operatorname{Im} \mu_{0}>0$ (it can be shown that there is only one such root). The symbol $\Gamma$ means integration over a halfcircle of the radius $|\mu|=1$ in the upper half-plane corresponding to the complex variable $\mu$. The neglect of the integral over $\Gamma$ in (21) is justified if $M \ll\left|\chi_{2} q\left(\mu_{0}\right)\right|$ where $M=\max \left|q(\mu)\left(1-\mu^{2}\right)\right|$ on $\Gamma$. This condition is satisfied for the isotropic turbulence $(q=$ const.) and also for a quite general class of anisotropic turbulence spectra. In the following we use, for $\omega \gg \omega_{c i}$, the estimate (21), thus conjecturing that the real turbulence spectra belong to this class.

The parameter $I$ in (21) is evidently an anisotropy factor: $I=1$ for isotropic turbulence $(q=$ const.) and $I \gg 1$ if the density fluctuations are greatly extended along the magnetic field $\mathbf{B}_{0}$. Thus, the density fluctuation function $q(\theta, \phi)$ is replaced in the tensor $\sigma_{t}$ by the two parameters $(\Delta n / n)^{2}$ and $I$, which can be found experimentally.

The integral $\mathscr{I}_{2}$ in (20) can be evaluated using the assumption

$$
\int_{0}^{\pi} q d \cos \theta /\left(1+\chi_{2}^{2} \cos ^{2} \theta\right) \simeq \chi_{2}^{-2} \simeq 0
$$

and the normalization (16):

$$
\mathscr{I}_{2}=2 \pi \int_{0}^{\pi} \frac{q \chi_{2}^{2} \cos ^{2} \theta d \cos \theta}{1+\chi_{2}^{2} \cos ^{2} \theta}=2 \pi \int_{0}^{\pi} q d \cos \theta-2 \pi \int_{0}^{\pi} \frac{q d \cos \theta}{1+\chi_{2}^{2} \cos ^{2} \theta} \simeq 1 .
$$

According to this estimate the turbulent current $j_{t z}$ directed along the applied magnetic field $\mathbf{B}_{0}$ is of the order of $(\Delta n / n)^{2}$ and hence will be neglected.

We can now rewrite the elements of the tensor (19) under the assumption $\omega \gg \omega_{c i}$ using the estimate (21) in the form

$$
\begin{aligned}
& \epsilon=1-\frac{\omega_{p e}^{2} Q}{\omega_{c e}^{2} \omega^{2}} A, \quad g=-\frac{\omega_{p e}^{2}}{\omega_{c e} \omega}, \quad \eta=1-\frac{\omega_{p e}^{2}}{\omega\left(\omega+i \nu_{e}\right)}, \\
& A=1-\left(\frac{\Delta n}{n}\right)^{2} \frac{\pi}{4} I \frac{\omega_{c e} \omega\left[\omega\left(\omega+i \nu_{e}\right)\right]^{\frac{1}{2}}}{Q^{\frac{3}{2}}} .
\end{aligned}
$$

This form of the conductivity tensor was used by Vaucher et al. (1982).

To gain some physical insight, we split this tensor into an electronic part $\epsilon_{e}$ and an ionic part $\epsilon_{i}:\left(\omega_{p i}^{2}=4 \pi e^{2} n / m_{i}\right)$

$$
\boldsymbol{\epsilon}=\boldsymbol{\epsilon}_{e}+\boldsymbol{\epsilon}_{i}, \quad \boldsymbol{\epsilon}_{e}=\left(\begin{array}{ccc}
\epsilon_{e} & i g & 0 \\
-i g & \epsilon_{e} & 0 \\
0 & 0 & \eta
\end{array}\right), \quad \boldsymbol{\epsilon}_{i}=-\mathbf{I} \frac{\omega_{p i}^{2}}{\omega^{2}}
$$


with

$$
\epsilon_{e}=1+\frac{\omega_{p e}^{2}\left(\omega+i \nu_{e}\right)}{\omega_{c e}^{2} \omega}\left(1+\frac{\pi}{4}\left(\frac{\Delta n}{n}\right)^{2} I\left(\frac{\omega}{\omega+i \nu_{e}}\right)^{\frac{1}{2}} \frac{\omega_{c e}}{Q^{\frac{1}{2}}}\right)
$$

The tensor $\epsilon_{e}$ is modified for directions perpendicular to the magnetic field $\mathbf{B}_{0}$ by the density fluctuations, while the tensor $\epsilon_{i}$ corresponding to the ionic part of the conductivity remains unaffected by the density fluctuations. However the ions influence indirectly the electronic part $\epsilon_{e}$ by virtue of the quasi-neutrality of the plasma. Note that the term $(\Delta n / n)^{2} I \omega_{c e} / Q^{\frac{1}{2}}$ in (25) can be very large, even for $(\Delta n / n)^{2} \ll 1$, since $I \gg 1$ and $\omega_{c e} /\left|Q^{\frac{1}{2}}\right| \gg 1$. It means that the influence of the density fluctuation on the electrical conductivity can be important in spite of the smallness of $(\Delta n / n)^{2}$.

\section{Propagation of EM waves in turbulent plasmas transverse to the magnetic field $B_{0}$}

The propagation of a plane EMW in an infinite plasma transverse to the applied magnetic field $\mathbf{B}_{0}$ (i.e. in the $r$ direction) is known to be characterized by the squared complex index of refraction

$$
\epsilon_{\perp}=c^{2} k^{2} / \omega^{2}=\left(\epsilon^{2}-g^{2}\right) / \epsilon, \text { where } \mathbf{E}, \mathbf{B} \propto \exp \left(i \omega c^{-1} \epsilon_{\perp}^{\frac{1}{2}} r\right) .
$$

Using the tensor (19) and neglecting the displacement current (i.e.

$$
\left.\left|\omega_{p e}^{2} Q A /\left(Q^{2}-P^{2}\right)\right| \gg 1\right)
$$

we can simplify $\epsilon_{\perp}$ as follows:

$$
\epsilon_{\perp}=\frac{\omega_{p e}^{2}}{Q A}=\frac{\omega_{p e}^{2}}{\omega_{h}^{2}} \frac{1}{\left(1-\omega\left(\omega+i \nu_{e}\right) / \omega_{h}^{2}\right)\left(1-(\Delta n / n)^{2} \mathscr{I}_{1}\left(P^{2} / Q^{2}+1\right)\right)},
$$

where $\omega_{p e}^{2} / \omega_{h}^{2}=c^{2} / c_{A}^{2}, c_{A}=B_{0} /\left(4 \pi n m_{i}\right)^{\frac{1}{2}}$ is the Alfvén velocity. Omitting the term proportional to $(\Delta n / n)^{2}$ we get the well-known expression

$$
\epsilon_{\perp}=\left(c^{2} / c_{A}^{2}\right)\left(1-\omega\left(\omega+i \nu_{e}\right) / \omega_{h}^{2}\right)^{-1}
$$

for the squared complex index of refraction. This expression corresponds to magnetosonic waves in a quiescent plasma, valid for $\omega \ll \omega_{c e}$. The presence of the term proportional to $(\Delta n / n)^{2}$ in (26) can lead to substantial changes of the real and imaginary part of $\epsilon_{\perp}$ and, thus, to new and important effects.

We shall now discuss $\epsilon_{\perp}$ (equation (26)) for the two cases $\omega_{c i} \ll \omega \ll \omega_{c e}$ and $\omega \lesssim \omega_{c i}$.

For $\omega \geqslant \omega_{c i}$ we can represent $\epsilon_{\perp}$, making use of (23), in alternative forms where

$$
\epsilon_{\perp}=\frac{c^{2}}{c_{A}^{2}} \frac{1}{1-i c^{2} \omega / 4 \pi \sigma_{\perp} c_{A}^{2}}=\frac{c^{2}}{c_{A}^{2}} \frac{1-\left(\omega^{2} / \omega_{h}^{2}\right)(1+\Delta \operatorname{Re}(\xi))+i \omega \nu_{\mathrm{eft}} / \omega_{h}^{2}}{\left(1-\left(\omega^{2} / \omega_{h}^{2}\right)(1+\Delta \operatorname{Re}(\xi))^{2}+\left(\omega \nu_{\mathrm{eft}} / \omega_{h}^{2}\right)^{2}\right.},
$$

$$
\begin{gathered}
\nu_{\mathrm{elf}}=\nu_{e}+\Delta \omega \operatorname{Im}(\xi), \quad \Delta=\frac{\pi}{4}\left(\frac{\Delta n}{n}\right)^{2} I\left(\frac{m_{i}}{m_{e}}\right)^{\frac{1}{2}}, \quad \xi=\left(\frac{\omega_{h}^{2}\left(\omega+i \nu_{e}\right)}{\omega Q}\right)^{\frac{1}{2}} \\
\sigma_{\perp}=\frac{\omega_{p e}^{2}}{4 \pi\left(\nu_{e}-i \omega\right) A_{e}}, \quad A_{e}=1+\frac{\pi}{4}\left(\frac{\Delta n}{n}\right)^{2} I\left(\frac{\omega_{c e}^{2} \omega}{\left(\omega+i \nu_{e}\right) Q}\right)^{\frac{2}{2}}
\end{gathered}
$$

The quantity $\sigma_{\perp}$ is the effective electronic transverse conductivity. Owing to the factor $1 / A_{e}$ this conductivity can be strongly reduced by the density fluctuations. 

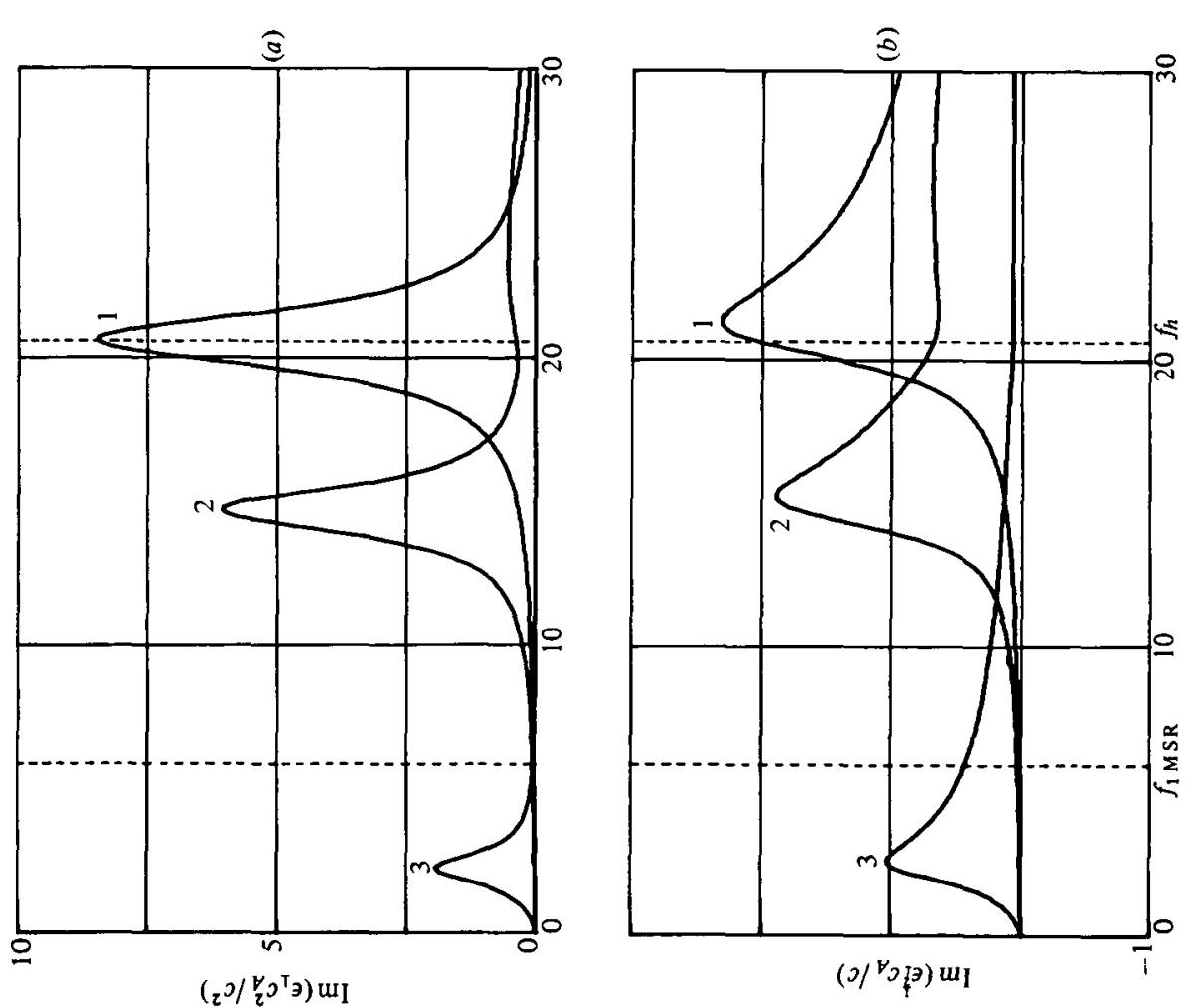

茝

屯

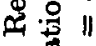

氙 उं 密

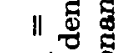
조

-

خे

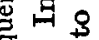

군

$\left.{ }^{-1}\right)^{8}$

\% 苍

용ㅇㅇㅇ

\&

몽

대용 का

a

$8-4$

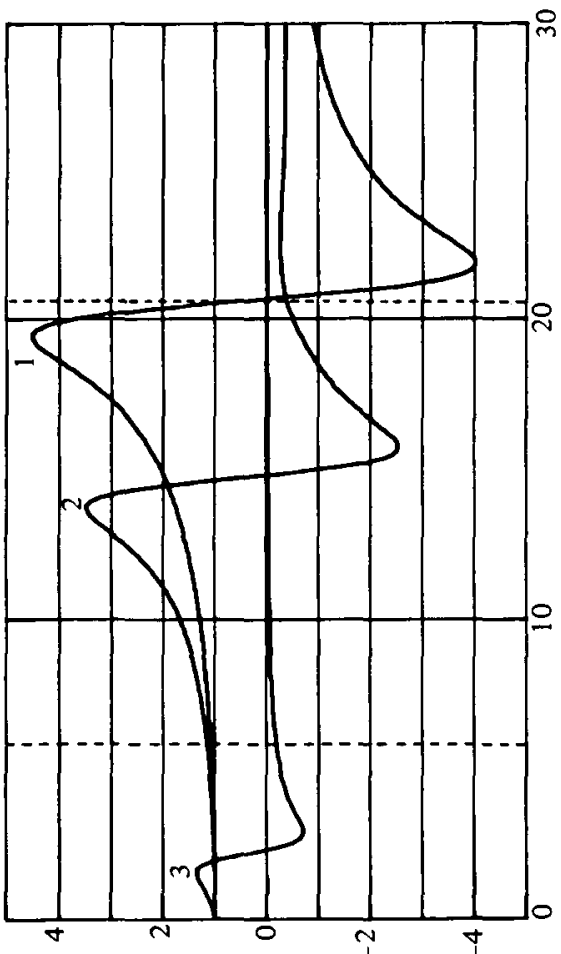

$\left.(\tau) / \tau^{\forall} \partial^{\top} \ni\right)$ วष

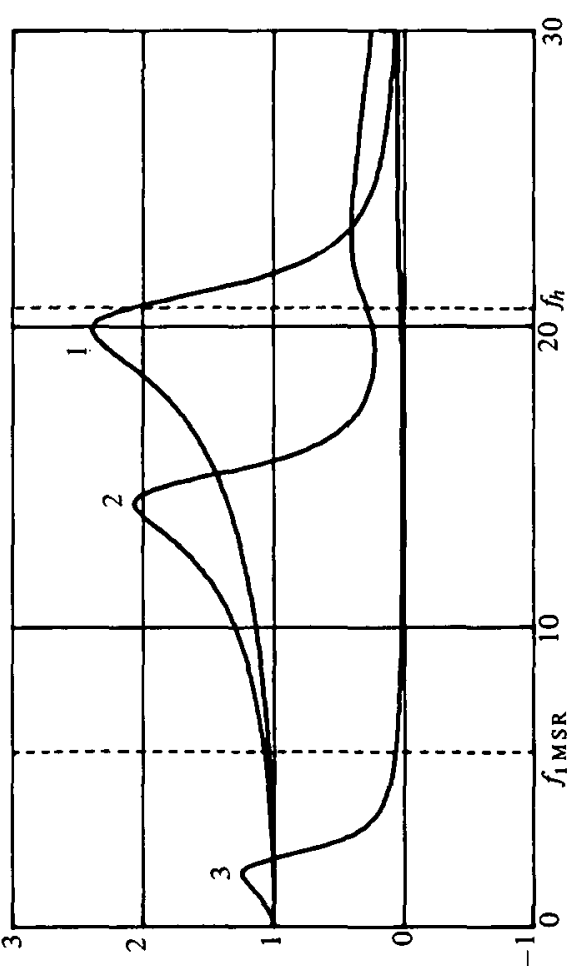

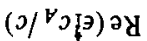

总

용

율

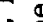

है

3

$\$$ 运

3110

㟧

(ิ)

¿ ${ }^{2} \dot{0}$

क t

¿

吉.

造 क⿺辶

严焉

点

馬

동

क⿺

象 0

ค 웜

बा प्र

兒

点 
$\nu_{\text {eff }}$ is the effective collision frequency which is increased, as compared with $\nu_{e}$, by the density fluctuations. For $\omega_{c i} \ll \omega \ll \omega_{c e}$ this enhancement is given by $\nu_{\text {eff }} / \nu_{e}=1+\Delta \simeq \Delta \gg 1$ if $\Delta \gg 1$. On the other hand, for $\omega \ll \nu_{e}, \omega \nu_{e} \gg \omega_{h}^{2}$ we get in accordance with the estimate (2) $\nu_{\text {eff }} / \nu_{e} \simeq \frac{1}{4} \pi(\Delta n / n)^{2} I \omega_{c e} / \nu_{e}$. As follows from (27), the fluctuations can affect the dielectric constant $\epsilon_{\perp}$ also in the limit $\nu_{e} \rightarrow 0$, since for $\Delta \operatorname{Re}(\xi) \gg 1$ they lead to a strong shift of the cut-off frequency defined by the equation $(1+\Delta \operatorname{Re}(\xi)) \omega^{2} / \omega_{h}^{2}=1$.

Turning now to the frequency diapason $\omega \lesssim \omega_{c i}$, we find from (19) that $\left|\mathscr{I}_{1}\right| \simeq 1, P /|Q| \lesssim 1$ must hold. Thus, the term due to the fluctuations in (26) appears to be of the order of $(\Delta n / n)^{2}$, i.e. its influence must be negligible. This conclusion can be interpreted as follows. For $\omega \lesssim \omega_{c i}$ the drift motion of the ions is influenced strongly by the applied magnetic field $\mathbf{B}_{0}$. Both electronic and ionic turbulent drift currents arise now under the influence of the density fluctuations. The ion turbulent drift current is directed opposite to the turbulent drift current of the electrons, shown in figure 1, and hence these currents compensate each other. Thus the strong electrodynamical effects due to the density fluctuations must disappear. It means that the turbulent plasma becomes transparent to the MSW for $\omega \lesssim \omega_{c i}$.

These results are illustrated in figure 2 where the values of $\operatorname{Re}\left(\epsilon_{\perp}\right), \operatorname{Im}\left(\epsilon_{\perp}\right)$, $\operatorname{Re}\left(\epsilon_{\perp}^{\frac{1}{2}}\right), \operatorname{Im}\left(\epsilon_{\perp}^{\frac{1}{2}}\right)$ as given by $(27)$ are plotted against $f=\omega /(2 \pi)$ for an argon plasma. We have assumed in figure 2 that the plasma is homogeneous and ascribed to it the same parameters of turbulence as those measured by Ritz et al. (1982) for the nonhomogeneous argon plasma: $\langle n\rangle=1.5 \times 10^{12} \mathrm{~cm}^{-3},\left\langle T_{e}\right\rangle=2.5 \mathrm{eV}, T_{i}=0.3 \mathrm{eV}$, $p_{0}=0.3 \mathrm{mTorr}, B_{0}=2 \mathrm{kG}, f_{c e}=5.4 \mathrm{GHz}, f_{c i}=73 \mathrm{kHz} .\langle n\rangle,\left\langle T_{e}\right\rangle$ are the electron density and temperature averaged over the radius. (It appears that these parameters are typical for the turbulence due to the drift instabilities.) The calculations presented in figure 2 were performed with a model-type fluctuation spectrum $\left|n_{\mathrm{r}}^{\prime}\right|^{2}=(4 \pi)^{-1}(\Delta n / n)^{2} \lambda_{r}^{2} \lambda_{z} \exp \left(-\lambda_{r} k_{r}-\lambda_{z}\left|k_{z}\right|\right)$ under the assumption $\lambda_{z} / \lambda_{r} \gg 1$. (The anisotropy factor $I$ introduced in (21) is equal to $I=2 \lambda_{z} / \lambda_{r}$ according to this model). It is evident from figure 2 that the presence of small density fluctuations in strongly magnetized plasmas can lead to a drastic change of the refractive index $\operatorname{Re} \epsilon_{\perp}$ and of the absorption coefficient $\operatorname{Im} \epsilon_{\perp}^{\frac{1}{2}}$. It is seen, in particular, that under the influence of the density fluctuations the maximum of the absoprtion coefficient $\operatorname{Im} \epsilon_{\perp}^{\frac{1}{2}}$ (which for $\Delta n / n=0$ is situated at $\omega \simeq \omega_{h}$ ) shifts to lower frequencies and in the region of our first magnetosonic resonance (1.MSR) strongly exceeds the absorption coefficient corresponding to the quiescent plasma. In the region of the lower-hybrid frequency $\omega_{h}$ and above it the damping by turbulence appears to be, in contrast, not so strong as for the quiescent plasma.

In the case of standing MSW excited in cylindrical plasmas of radius $a$, magnetized by an axial magnetic field $B_{0}$, the radial distributions of the EM field components $E_{\phi}$ (azimuthal electric component) and $B_{z}$ (axial magnetic component) are described for an infinitely long cylindrical plasma and an infinitely long exciting coil by the equations

$$
\frac{d}{d r} \frac{1}{r} \frac{d}{d r} r E_{\phi}+\frac{\omega^{2}}{c^{2}} \epsilon_{\perp} E_{\phi}=0, \quad i \frac{\omega}{c} B_{z}=\frac{1}{r} \frac{d}{d r} r E_{\phi}
$$




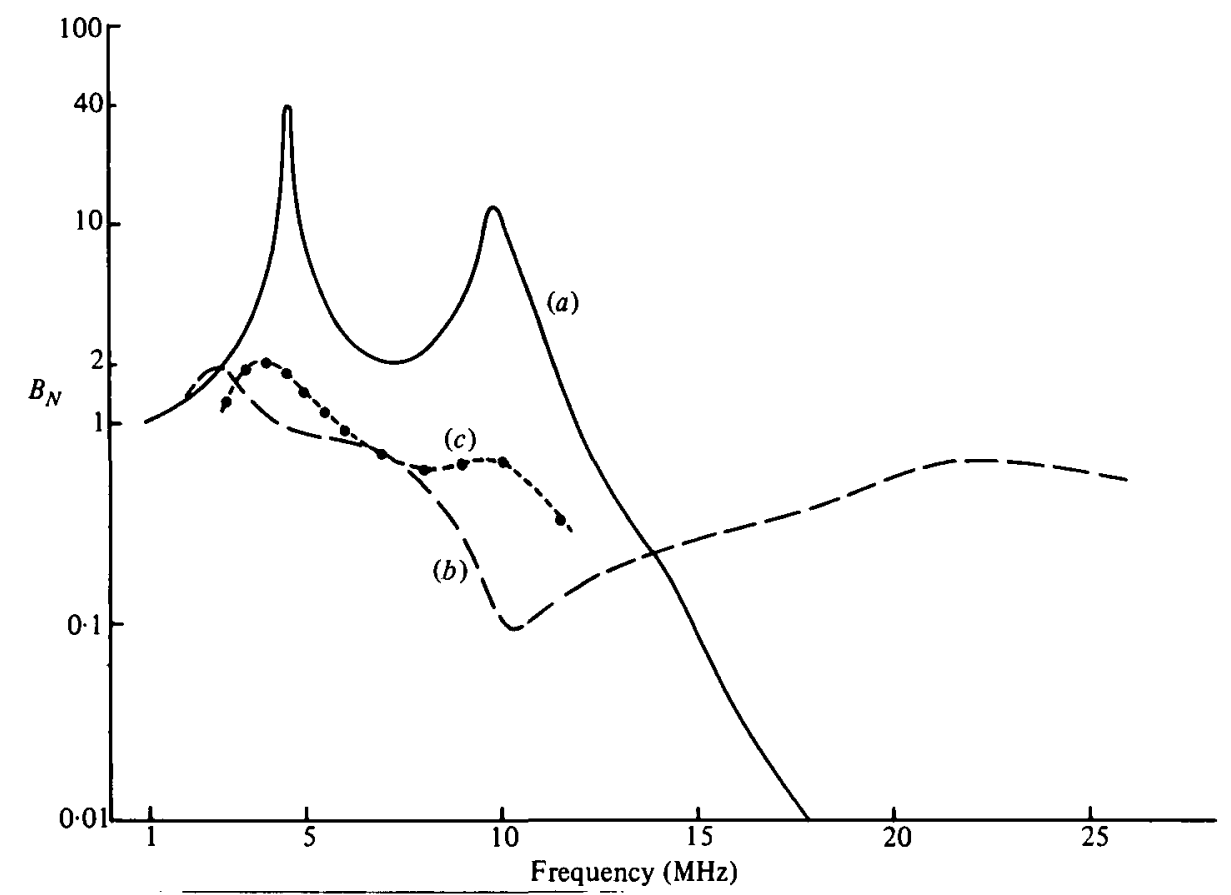

Figure 3. Normalized amplitude $B_{N}=\left|B_{z}(0) / B_{z}(a)\right|$ for an inhomogeneous argon plasma. Theoretical calculations: $(a)$ plasma without fluctuation, $(b)$ with fluctuation $(\Delta n / n)_{\max }=$ $11 \%$ (other parameters are the same as in figure 2). (c) Experiment. (For additional details, see Ritz et al. (1982).)

As boundary condition we use the requirement that $B_{z}(a)$ is equal to the vacuum field.

In the experiments the quantities $B_{N}$ and $\phi$, defined by

$$
B_{z}(0) / B_{z}(a)=B_{N} \exp (i \phi),
$$

were measured. A quantitative comparison of the experimental (Ritz et al. 1982) and theoretical values of $B_{N}(\omega)$ and $\phi(\omega)$ for the plasma characterized by the distributions $\left[n(r),(\Delta n / n)(r), T_{e}(r)\right]$ is obtained by solving (28) numerically. This calculation was performed with a code written by Räuchle (1972). The result is presented in figure 3. As it shows, the theory provides a satisfactory quantitative explanation of the drastic discrepancies between experiment and theory for a quiescent plasma. Further experiments and new calculations for a plasma of finite length (Vaucher et al. 1983) confirm this agreement.

In the experiments with hydrogen plasmas (Lammers 1974; Krämer 1975) the plasma admittance $1 / R$, allowing the power $P$ absorbed in the plasma to be expressed by the formula

$$
P=|U|^{2} /(2 R), \quad\left(U=2 \pi a E_{\phi}(a) \text { is the voltage }\right)
$$

was measured. For a long cylindrical plasma of length $L(L \gg a)$, excited by a coil of the same length, the plasma admittance can be expressed through the dimensionless surface impedance (as defined in Landau \& Lifshitz 1960, §67), 


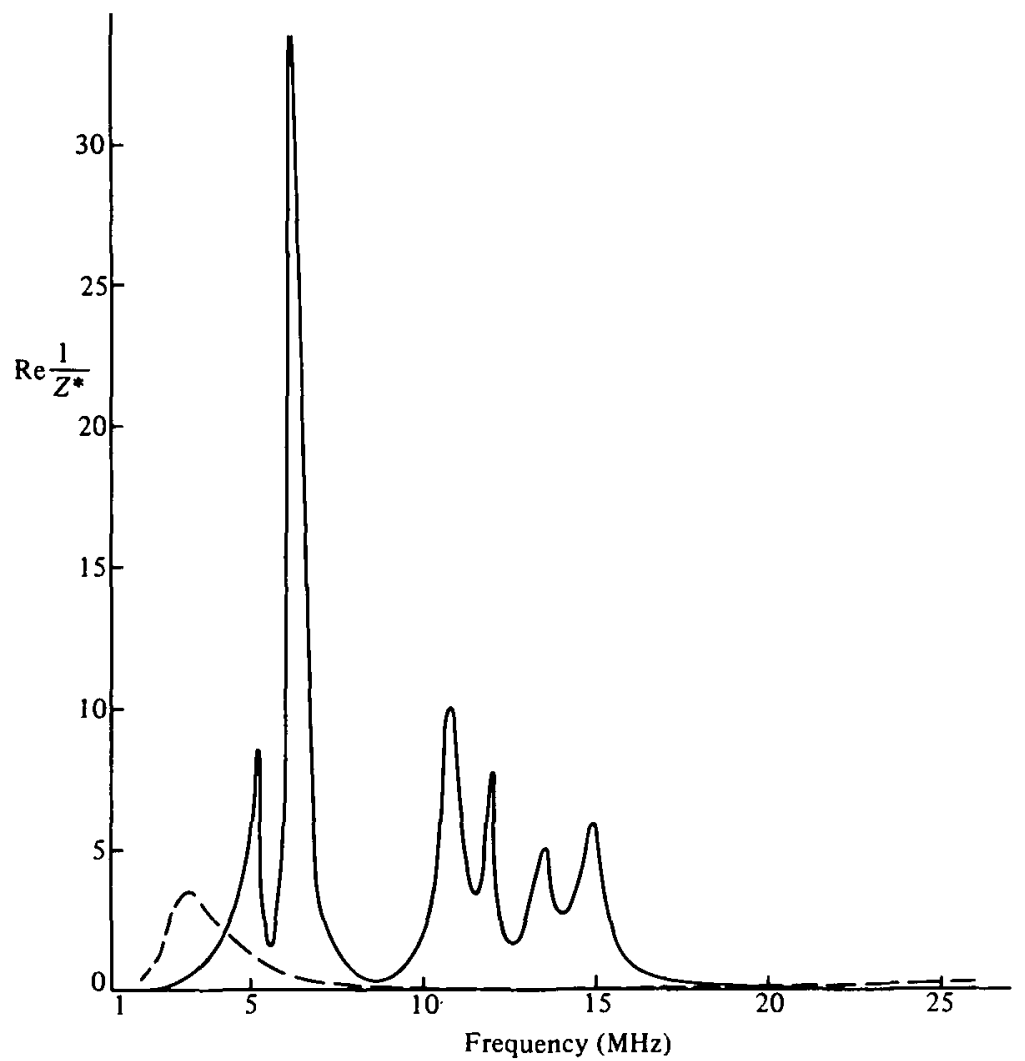

FIGURE 4. Calculated plasma admittance $\operatorname{Re}_{\theta}\left(1 / Z^{*}\right)$ of the inhomogeneous cylindrical argon plasma. - : plasma without density fluctuation, - - - : with density fluctuation.

$Z=E_{\phi}(a) / B_{z}(a)$ by the formula $1 / R=c L /\left(8 \pi^{2} a\right) \operatorname{Re}\left(1 / Z^{*}\right)$. Krämer (1975, table 1) found that the maximal values of the plasma admittance are lower by a factor 0.1-0.01 than those following from the quiescent plasma theory. Direct comparison of the present theory with these experiments is impossible since the parameters of the hydrogen plasma turbulence were not measured. However, we performed calculations of the admittance of the argon plasma investigated by Ritz et al. (1982) under the same assumptions as those explained in figure 3. Results of these calculations presented in figure 4 show that the turbulent plasma admittance proves to be, in general, much lower than the corresponding quantity following from the quiescent plasma theory. As a crude estimate shows, the maximal plasma admittance for the parameters of the turbulence in this radially inhomogeneous plasma is lower by a factor $1 /\left[(\Delta n / n)_{\max }^{2}(\Delta r / a) I\left(m_{i} / m_{e}\right)^{\frac{1}{2}}\right] \simeq 0 \cdot 1$ than expected by the classical theory. $\left((\Delta n / n)_{\max }^{2}\right.$ is the maximal value of the relative density fluctuation, $\Delta r$ is the thickness of the sheath in which the fluctuations occur). Moreover, the dependence $1 / R$ on the frequency $\omega$ (figure 4 ) does not show a pronounced periodic structure. This is again in contrast to the quiescent plasma theory and in accordance with the measurements of Lammers (1974). Thus, it appears that including the density fluctuations allows us to explain qualitatively the lowering of the plasma admittance in such experiments. 


\section{Propagation of EM waves in turbulent plasmas parallel to the magnetic field $B_{0}$}

In the case of a plane wave $\mathbf{E}, \mathbf{B} \propto \exp \left(i \omega c^{-1} \epsilon_{\|}^{\frac{1}{2}} z\right)$ propagating in an infinite plasma parallel to the magnetic field $\mathbf{B}_{0}$ ( $z$ direction) the effective dielectric constant $\epsilon_{\|}$is given by $\epsilon_{\|}=\epsilon \mp g$. We restrict ourselves to the case of electron whistlers $(\epsilon=\epsilon-g)$ and to the frequency interval $\omega_{c i} \ll \omega<\omega_{h} \ll \omega_{c e}$ with the assumption $\nu_{e} \ll \omega$. The refractive index $\operatorname{Re} \epsilon_{\|}^{\frac{1}{2}}$ and the absorption coefficient $\kappa=\operatorname{Im} \epsilon_{\|}^{\frac{1}{2}}$ are given now according to (23) by the formulae:

$$
\operatorname{Re} \epsilon_{\|}^{\frac{1}{2}}=\left(\frac{\omega_{p e}^{2}}{\omega \omega_{c e}}\right)^{\frac{1}{2}}, \quad \kappa=\left(\frac{\omega_{p e}^{2}}{\omega \omega_{c e}}\right)^{\frac{1}{2}} \frac{\nu_{e}}{2 \omega_{c e}}(1+\Delta), \quad \Delta=\frac{\pi}{4}\left(\frac{\Delta n}{n}\right)^{2} I\left(\frac{m_{i}}{m_{e}}\right)^{\frac{1}{2}} .
$$

As follows from (29), the presence of the density fluctuations is equivalent to an increase of the collision frequency $\nu_{c}$ by the factor $1+\Delta$ which can be very big if the fluctuation level $\Delta n / n$ is high enough.

It is tempting to apply these considerations to ionospheric whistlers, which often disappear for reasons which are not yet well known. Assuming that a whistler propagates in the ionosphere perpendicular to the Earth's surface and nearly parallel to the line of force of the Earth's magnetic field, we can characterize the damping of the whistlers in the ionosphere by the quantity

$$
\gamma=\omega c^{-1} \int_{0}^{h_{0}} \kappa d h .
$$

$h$ is the height measured from the Earth's surface and $h_{0} \simeq 10^{3} \mathrm{~km}$. The factor $\Delta$ in (29) can be estimated roughly assuming $\Delta n / n=0 \cdot 1$ (this is a plausible value for the disturbed $\mathrm{D}, \mathrm{E}$ and $\mathrm{F}$ ionospheric layers according to table 5.2 in $\mathrm{Al}$ 'pert (1972)), $I=50$ (the fluctuations are highly extended along the lines of force of the Earth's magnetic field, see Rawer \& Suchy (1967, p. 355)), $\left(m_{i} / m_{e}\right)^{\frac{1}{2}} \simeq 200$. We get in this way $\Delta \simeq 100$. Thus, the absorption coefficient $\kappa$ increases by a factor of 100 owing to the density fluctuations in this case. Taking the average values $\nu_{e}=10^{3} \mathrm{sec}^{-1}, \omega_{p e}=5 \times 10^{7} \mathrm{sec}^{-1}, \omega_{c e}=7 \times 10^{6} \mathrm{sec}^{-1}$ according to table 3 in Ginzburg \& Ruhadze (1975) and a typical whistler frequency $\omega=2 \times 10^{4} \mathrm{sec}^{-1}$, we can estimate the damping of the whistlers. For a relatively quiescent ionospheric plasma $(\Delta n / n=0 \cdot 01-0.001)$ we get $\gamma \simeq 0.5$. In this case the whistlers will not be damped very much during the passage through the ionosphere. If we assume, however, that the absorption coefficient $\kappa$ increases under the influence of stronger turbulences $(\Delta n / n \simeq 0 \cdot 1)$ by a factor $\Delta=100$, we get $\gamma \simeq 50$. Such whistlers will be completely damped in the ionosphere.

Thus, this preliminary estimate seems to confirm a conjecture formulated first by Budden (1959) that the disappearance of the whistlers may be due to the increase of the density fluctuations in the ionosphere.

\section{Conclusion}

We have performed a general investigation of some qualitatively new electrodynamical phenomena arising in strongly magnetized plasmas under the influence of small density fluctuations. The basic idea is that the presence of 
such fluctuations can lead to a strong redistribution of the electric currents provoked by the RF electric field $\mathbf{E}$.

In a quiescent strongly magnetized plasma the electrons perform mainly a drift motion perpendicular to the static magnetic field $\mathbf{B}_{\mathbf{0}}$ and to the RF field $\mathbf{E}$. The currents $\mathbf{j}$ directed along the $\mathbf{E}$ field and the electromagnetic energy transfer to the plasma $\mathbf{j}$. $\mathbf{E}$ are small in this case. The appearance of stochastic fields $\mathbf{E}^{\prime}$, directed perpendicular to $\mathbf{B}_{0}$ and $\mathbf{E}$, due to density fluctuations, provokes strong drift currents $\mathbf{j}_{t}$ flowing along $\left[\mathbf{E}^{\prime} \times \mathbf{B}_{0}\right]$, i.e. along $\mathbf{E}$. Owing to these drift currents the EM energy transfer $\mathbf{j}_{t}$. $\mathbf{E}$ to the plasma may be strongly increased.

Under the basic assumptions that the density fluctuations are not affected by the applied EM field and that their space scales are small, we were able to derive a general expression for the electrical conductivity tensor for such plasmas $((17),(23))$. This theory allows us to interpret in a natural way results of several experiments, in which anomalously strong damping of the magnetosonic waves in turbulent strongly magnetized plasmas was observed ( $\$ 3)$. The theory has also a number of other applications, as demonstrated in $\$ 4$ for the example of ionospheric whistlers.

We would like to express our appreciation to Dr E. Räuchle, University of Stuttgart, who kindly made a computer program available to us. This work was supported by the Swiss National Science Foundation.

\section{Appendix A}

To estimate errors involved in the quasi-electrostatic approximation (replacement of the equation curl $\mathbf{E}^{\prime}=i(\omega / c) \mathbf{B}^{\prime}$ in $(8)$ by curl $\mathbf{E}^{\prime}=0$ ) we will represent the fluctuating field components $\mathbf{E}^{\prime}, \mathbf{B}^{\prime}$ in the form $\mathbf{E}^{\prime}=\mathbf{E}_{0}^{\prime}+\mathbf{E}_{1}^{\prime}, \mathbf{B}^{\prime}=\mathbf{B}_{0}^{\prime}+\mathbf{B}_{1}^{\prime}$. Here $\mathbf{E}_{\mathbf{0}}^{\prime}, \mathbf{B}_{\mathbf{0}}^{\prime}$ correspond to the quasi-electrostatic approximation (which will use under conditions allowing us to neglect the displacement current). They are governed by the equations

$$
\operatorname{curl} \mathbf{E}_{0}^{\prime}=0, \quad \operatorname{curl} \mathbf{B}_{0}^{\prime}=\frac{4 \pi}{c} \sigma \mathscr{E}^{\prime}, \quad \mathscr{E}^{\prime}=-\nabla \phi^{\prime}+\frac{n^{\prime}}{n} \mathbf{E} .
$$

$\mathbf{E}_{1}^{\prime}$ and $\mathbf{B}_{1}^{\prime}$ are corrections to this approximation. The vector $\mathbf{E}_{1}^{\prime}$ satisfies the equation curl $\mathbf{E}_{1}^{\prime}=i(\omega / c) \mathbf{B}_{0}^{\prime}$. Using the Fourier representations

$$
\mathbf{E}_{1}^{\prime}=\sum_{\mathbf{k}} \mathbf{E}_{1 \mathbf{k}}^{\prime} e^{i \mathbf{k} \cdot \mathbf{r}}, \quad \mathbf{B}_{0}^{\prime}=\sum_{\mathbf{k}} \mathbf{B}_{0 \mathbf{k}}^{\prime} e^{i \mathbf{k} \cdot \mathbf{r}}
$$

we get the following equations for the Fourier coefficients $\mathbf{E}_{1 \mathbf{k}}^{\prime}, \mathbf{B}_{\mathbf{0 k}}^{\prime}$ :

$$
\left[\mathbf{k} \times \mathbf{E}_{1 k}^{\prime}\right]=\frac{\omega}{c} \mathbf{B}_{0 \mathbf{k}}^{\prime}, \quad\left[\mathbf{k} \times \mathbf{B}_{0 \mathbf{k}}^{\prime}\right]=-\frac{4 \pi i}{c} \sigma \mathscr{E}_{\mathbf{k}}^{\prime}, \quad \mathscr{E}_{k}^{\prime}=-i \mathbf{k} \phi_{\mathbf{k}}^{\prime}+n_{\mathbf{k}}^{\prime} \cdot \mathbf{E}
$$

They provide a solution

$$
\mathbf{E}_{1 \mathbf{k}}^{\prime}=\frac{4 \pi i \omega}{c^{2} k^{2}}\left(\sigma \mathscr{E}_{\mathbf{k}}^{\prime}-\mathbf{e}\left(\mathbf{e} \sigma \mathscr{E}_{\mathbf{k}}^{\prime}\right)\right), \quad \mathrm{e}=\frac{\mathbf{k}}{k}
$$


for the coefficient $\mathbf{E}_{\mathbf{1}}^{\prime}$. According to (10) the corresponding correction $\delta \mathbf{j}_{t}$ to the turbulent drift current is

$$
\delta \mathbf{j}_{t} \simeq \sigma_{0} \sum_{\mathbf{k}} n_{\mathbf{k}}^{\prime *} \mathbf{E}_{\mathbf{l}}^{\prime}=\frac{4 \pi i \omega \sigma_{0}}{c^{2}} \int \frac{1}{k^{2}}\left(\sigma n_{\mathbf{k}}^{\prime *} \mathscr{E}_{\mathbf{r}}^{\prime}-\mathbf{e}\left(\mathbf{e} \cdot \sigma n_{\mathbf{k}}^{\prime *} \mathscr{E}_{\mathbf{k}}^{\prime}\right)\right) d \mathbf{k}
$$

If the electric field $\overline{\mathbf{E}}$ possesses only components perpendicular to the static magnetic field $\mathbf{B}_{\mathbf{0}}$ (as is the case for cylindrical MSW investigated in $\$ 3$ ), the correction $\delta \mathbf{j}_{t}$ can be estimated via (A 4), under the same assumptions as those leading to the tensor (23), in the form

$$
\left|\delta \mathbf{j}_{t}\right| \simeq \sigma_{0} \frac{\omega}{c^{2}} \frac{\omega_{p e}^{2}}{\omega_{c e}} \lambda_{r}^{2}\left(\frac{\Delta n}{n}\right)^{2}|\mathbf{E}|
$$

Here $\lambda_{r}$ is an effective length of the density fluctuation in the direction perpendicular to the magnetic field $\mathbf{B}_{0}$. Comparison of (A 5) and of (11) gives

$$
\left|\delta \mathbf{j}_{t} / \mathbf{j}_{t}\right| \simeq \lambda_{r}^{2} / \delta^{2}, \quad \delta=\left(c / \omega_{p e}\right)\left(\omega_{c e} / \omega\right)^{\frac{1}{2}}
$$

Under the conditions assumed in $\S 3$ this quantity is always very small.

\section{Appendix B}

Exploiting the symmetry properties of the angular distribution $q(\theta, \phi)$ assumed in the text $\left(q(\theta, \phi)\right.$ is $\phi$-independent and $\left.q(\theta)=q\left(\frac{1}{2} \pi-\theta\right)\right)$ we can bring the tensor $A$ in (15) to the form

$$
\mathbf{A}=\left(\begin{array}{ccc}
-\mathscr{I}_{1} & -\chi_{1} \mathscr{I}_{1} & 0 \\
\chi_{1} \mathscr{I}_{1} & -\mathscr{I}_{1} & 0 \\
0 & 0 & -\chi_{2}^{2} \mathscr{I}_{2}
\end{array}\right)
$$

where $\mathscr{I}_{1}, \mathscr{I}_{2}$ are the integrals defined in (19). The product of the tensors $\sigma(12)$ and $\mathbf{A}$ reduces now to

$$
\sigma \mathbf{A}=\left(\begin{array}{ccc}
\left(\sigma_{2} \chi_{1}-\sigma_{1}\right) \mathscr{I}_{1} & -\left(\sigma_{2}+\chi_{1} \sigma_{1}\right) \mathscr{I}_{1} & 0 \\
\left(\sigma_{2}+\chi_{1} \sigma_{1}\right) \mathscr{I}_{1} & \left(\sigma_{2} \chi_{1}-\sigma_{1}\right) \mathscr{I}_{1} & 0 \\
0 & 0 & -\sigma_{0} \chi_{2}^{2} \mathscr{I}_{2}
\end{array}\right)
$$

Using relation (14) $\chi_{1}=\sigma_{2} / \sigma_{1}=i P / Q$, we can rewrite the elements of the tensor (B 2) also in the form

$$
\left(\sigma_{2} \chi_{1}-\sigma_{1}\right)=-\sigma_{1}\left(P^{2} / Q^{2}+1\right) \mathscr{I}_{1}, \quad\left(\sigma_{2}+\chi_{1} \sigma_{1}\right) \mathscr{I}_{1}=2 \sigma_{2} \mathscr{J}_{1} .
$$

With (17) and (B 2) we can write the dielectric tensor (18) in the explicit form

$$
\boldsymbol{\epsilon}_{t}=\left(\begin{array}{ccc}
\epsilon & i \tilde{g} & 0 \\
-i \tilde{g} & \epsilon & 0 \\
0 & 0 & \eta
\end{array}\right), \quad \tilde{g}=g\left(1+2\left(\frac{\Delta n}{n}\right)^{2} \mathscr{I}_{1}\right),
$$

where the elements $\epsilon, \eta, g$ are defined in (19).

One must stress here the basic difference between the terms containing $(\Delta n / n)^{2}$ in the diagonal and in the off-diagonal elements of (B 3). In the former the parameter $(\Delta n / n)^{2}$ (which was assumed to be small from the very beginning) is multiplied by the factor $\left(P^{2} / Q^{2}+1\right)$ which remains very large in strongly 
magnetized plasmas for the frequences $\omega>\omega_{c i}$. This factor reaches values of the order of $\left(\omega_{c e} /\left(\omega+i v_{e}\right)\right)^{2}$ for $\omega>\omega_{h}$ or $\omega_{c e} / \omega_{c i}$ for $\omega \approx \omega_{h}$. In contrast, in the offdiagonal elements of (B 3) the parameter $(\Delta n / n)^{2}$ is multiplied by the factor $2 \mathscr{F}_{1}$, having the property $2\left|\mathscr{F}_{1}\right| \lesssim 1$. Thus the term containing $(\Delta n / n)^{2}$ in $\tilde{g}$ of (B 3) can always safely be neglected, as was done in the expression (19).

\section{REFERENCES}

AL'PERT, JA. L. 1972 Propagation of Electromagnetic Waves and the Ionosphere. Consultants Bureau.

Blackwell, B. D. \& Cross, R. C. 1979 J. Plasma Phys. 22, 499.

BUDdEN, K. G. 1959 J. Res. N.B.S., 63D, 135.

Ginzbura, V.L. 1961 Propagation of Electromagnetic Waves in Plasma. Gordon and Breach.

Ginzbura, V. L. \& Ruhadze, A. A. 1975 Waves in a Magneto-active Plasma (in Russian). Nauka.

Hoeqger, B. A., Ritz, Ch., Schneider, H. \& Vaucher, B. G. 1980 Phys. Lett. 76 A, 393.

Hoegger, B. A., Ritz, Сh., Schneider, H. \& Vaucher, B. G. 1981 Phys. Lett. 84 A, 250.

Kadomtsev, B. B. 1965 Plasma Turbulence. Academic.

Kratl, N. A. \& Trivelpiece, A. W. 1973 Principles of Plasma Physics. McGraw-Hill.

Krämer, M. 1975 Plasma Phys. 17, 373.

Lammers, B. 1974 Dissertation, Ruhr-Universität Bochum.

LandaU, L. D. \& Lifshitz, E. M. 1960 Electrodynamics of Continuous Media. AddisonWesley.

RÄUCHLE, E. 1972 Report IPF-72-8, Institut für Plasmaforschung, Universität Stuttgart.

RAWER, K. \& SUCHY, K. 1967 Encyclopedia of Physics, vol. 49/2. Springer.

Ritz, Ch., Hoegger, B. A., Sayasov, Yu. S., Schneider, H. \& Vaucher, B. G. 1982 Helv. Phys. Acta, 55, 354.

SaYasov, Yv. S. 1981 Phys. Lett. 82 A, 337.

Schneider, H., Hokgger, B. A., Ritz, Ch., Vaudher, B. G. \& Tran, T. M. 1980 Helv. Phys. Acta, 53, 40.

TER HAAR, D. 1958 Introduction to the Physics of Many-Body Systems, Appendix B. Interscience.

Trmofeev, A. V. \& Shvilkin, B. N. 1976 Soviet Phys. Uspekhi, 19, 149.

Vaucher, B. G., Hoegger, B. A., Ritz, Ch., Sayasov, Yu. S. \& Schneider, H. 1983 Plasma Phys. 25, 331.

Yoshikawa, S. \& Rose, D. J. 1962 Phys. Fluids, 5, 334. 